\title{
Synergistic antioxidant activity of resveratrol with genistein in high-glucose treated Madin-Darby canine kidney epithelial cells
}

\author{
CHISHIH CHU ${ }^{1}$, FUNG-JOU LU ${ }^{2}$, RANG-HUI YEH ${ }^{3}$, ZIH-LING LI $^{1}$ and CHING-HSEIN CHEN ${ }^{1}$ \\ ${ }^{1}$ Department of Microbiology, Immunology and Biopharmaceuticals, College of Life Sciences, \\ National Chiayi University, Chiayi 60004; ${ }^{2}$ Institute of Medicine, Chung Shan Medical University, Taichung 40201; \\ ${ }^{3}$ Department of Physical Medicine and Rehabilitation, Chia-Yi Chang Gung Memorial Hospital, Chiayi 61363, Taiwan, R.O.C.
}

Received November 17, 2015; Accepted December 16, 2015

DOI: $10.3892 /$ br.2016.573

\begin{abstract}
Resveratrol (Re), a stilbenoid, is associated with a potential benefit in controlling certain biomarkers in type II diabetes. Genistein (Ge), a phytoestrogen, may act as an antioxidant and thus may diminish damaging effects of free radicals in tissues. In the present study, a potential synergistic antioxidant effect of an Re/Ge combination on high-glucose (HG) incubation in Madin-Darby canine kidney (MDCK) epithelial cells was evaluated. Compared with the treatment of Re or Ge alone, the $\mathrm{Re} / \mathrm{Ge}$ combination synergistically decreased intracellular reactive oxygen species (ROS) and hydroxyl radicals in MDCK cells. This synergistic antioxidant effect correlated with the inhibition of nicotinamide adenine dinucleotide phosphate (NADPH) oxidase expression and an increase in $\gamma$-glutamylcysteine synthetase expression. In addition, mitochondrial complex I, NADPH oxidase, xanthine oxidase and lipoxygenase contributed towards ROS overproduction when the MDCK cells were incubated with HG. In conclusion, the Re/Ge combination synergistically enhanced the antioxidant effect in HG-incubated kidney cells, possibly through an enhanced antioxidant regulation mechanism. The Re/Ge combination may be a potential benefit against oxidative stress in diabetes mellitus.
\end{abstract}

\section{Introduction}

Diabetes mellitus is a metabolic disease in which blood sugar concentrations are high over a prolonged period. Without proper treatment, diabetes mellitus can trigger a number of complications, including chronic kidney failure (1). Diabetic nephropathy is a critical event in end-stage renal failure worldwide. Its morphological features include glomerular hypertrophy, mesangial

Correspondence to: Professor Ching-Hsein Chen, Department of Microbiology, Immunology and Biopharmaceuticals, College of Life Sciences, National Chiayi University, 300 Syuefu Road, Chiayi 60004, Taiwan, R.O.C.

E-mail: chench@mail.ncyu.edu.tw

Key words: resveratrol, genistein, antioxidant, Madin-Darby canine kidney cells, reactive oxygen species expansion, basement membrane thickening, interstitial fibrosis, tubular atrophy and arteriolar thickening (2). Numerous studies indicate that oxidative stress is a typical factor associated with the main pathways involved in the development and progression of diabetic microvascular and macrovascular complications of diabetes. There are a number of pathways that have been associated with increased production of intracellular reactive oxygen species (ROS), such as nicotinamide adenine dinucleotide phosphate (NADPH) oxidase, mitochondrial respiratory chain via oxidative phosphorylation, advanced glycation end products, defects in the polyol pathway and uncoupled nitric oxide synthase (2). Excess amounts of ROS directly damage cellular micromolecules and macromolecules, which eventually cause end-stage renal disease. Diminishing the production of ROS may be a favorable therapeutic treatment to improve renal damage from diabetic nephropathy.

Resveratrol $(\mathrm{Re})$ is found in various plants, including grapes, berries and peanuts. It is also present in wine, particularly red wine. Re has been the focus of numerous studies investigating its biological attributes, which include mainly antioxidant activities (3). Re is believed to have antioxidant properties, protecting the body against the type of damage associated with an increased risk for conditions, such as cancer and heart disease. As Re is believed to have numerous health benefits, a number of manufacturers have tried to capitalize by the production of Re supplements.

Isoflavones are able to neutralize free radicals. Among the isoflavones, genistein $(\mathrm{Ge})$ has the highest antioxidant activity. Ge may act as direct antioxidant, similar to a number of other isoflavones, and thus may alleviate the damaging effects of free radicals in tissues $(4,5)$. Ge is a strong antioxidant that eliminates damaging free radicals and reduces lipid peroxidation, and increases the activity of other antioxidant enzymes, such as glutathione peroxidase, superoxide dismutase and glutathione reductase (6).

These previous studies emphasize the importance of Re and $\mathrm{Ge}$ in antioxidant activity and cellular protection from ROS damage. However, to the best of our knowledge, there are no previous studies evaluating the synergistic effects of Re with Ge on the high-glucose (HG) treatment in kidney cells and its possible protective mechanisms. The present study established a HG treatment in an MDCK cell line and evaluated the possible synergistic antioxidant efficacy of Re/Ge combination 
treatment. Specifically, the sources of ROS generation from the HG incubation of MDCK cells were examined and the molecular mechanisms underlying synergistic antioxidant activity were determined.

\section{Materials and methods}

Cell line and reagents. The MDCK cell line was obtained from the Bioresource Collection and Research Center (Hsinchu, Taiwan). Dichlorofluorescein diacetate (DCFH-DA) was acquired from Invitrogen Co. (Carlsbad, CA, USA). The primary antibodies against p47phox, p22, $\gamma$-glutamylcysteine synthetase ( $\gamma$-GCS), catalase, superoxide dismutase-1 (SOD-1), glyceraldehyde 3-phosphate dehydrogenase (GAPDH) and secondary antibodies were obtained from Santa Cruz Biotechnology, Inc. (Santa Cruz, CA, USA). The primary antibodies against $\beta$-actin, hydroxyphenyl fluorescein (HPF) and other chemicals were purchased from Sigma-Aldrich (St. Louis, MO, USA).

Cell culture and treatment. MDCK $\left(1 \times 10^{6}\right)$ cells were cultured in a minimum essential medium (MEM) (90\%) supplemented with $2 \mathrm{mM} \mathrm{L}$-glutamine and Earle's BBS adjusted to contain $1.5 \mathrm{~g} / 1$ sodium bicarbonate, $0.1 \mathrm{mM}$ non-essential amino acids, $1.0 \mathrm{mM}$ sodium pyruvate, $10 \%$ fetal bovine serum, $100 \mathrm{U} / \mathrm{ml}$ penicillin and $100 \mu \mathrm{g} / \mathrm{ml}$ streptomycin in $100-\mathrm{mm}$ cultured dishes at $37^{\circ} \mathrm{C}$ in a humidified atmosphere of $5 \% \mathrm{CO}_{2}$. When cells reached $80 \%$ confluence in the 100 -mm cultured dishes, they were washed with phosphate-buffered saline (PBS) and trypsinized for use in various experiments. The MDCK cells were plated in $60-$ or $100-\mathrm{mm}$ culture dishes for $24 \mathrm{~h}$. The culture medium was replaced with five types of media: i) Normal glucose MEM medium (NG) containing $5.5 \mathrm{mM}$ glucose, ii) HG MEM medium (HG) containing $30 \mathrm{mM}$ glucose, iii) $3 \mu \mathrm{M}$ Re alone in HG medium, iv) $1 \mu \mathrm{M}$ Ge alone in HG medium and v) Re and Ge in HG medium for $48 \mathrm{~h}$. The selective inhibitors and the particular concentrations used to inhibit the intracellular ROS generation from various enzymes were referenced and modified from our previous study (7). The particular concentrations of the selective inhibitors did not affect the cell viability of MDCK cells. The selective inhibitor of mitochondrial complex I [ $20 \mathrm{nM}$ rotenone (RO)], mitochondrial complex II [5 $\mu \mathrm{M}$ carboxin (Car) or $5 \mu \mathrm{M}$ 2-ethenoyltrifluoroacetone (TTFA)], mitochondrial complex III [0.1 nM antimycin A (AA)], NADPH oxidase [2 $\mu \mathrm{M}$ 4-(2-aminoethyl)benzenesulfonyl fluoride hydrochloride (AEB) or $30 \mu \mathrm{M}$ apocynin (Apo)], xanthine oxidase [10 $\mu \mathrm{M}$ allopurinol (Allo)] and liopoxygenase [5 $\mu \mathrm{M}$ nordydihydroguaiaretic acid (Nordy)] were pretreated for $1 \mathrm{~h}$, followed by HG treatment for $48 \mathrm{~h}$. These inhibitors were purchased from Sigma-Aldrich.

Measurement of intracellular ROS by flow cytometry. Production of intracellular ROS was detected by flow cytometry using the DCFH-DA probe (Sigma-Aldrich). The MDCK $\left(2 \times 10^{5}\right)$ cells were plated in $60-\mathrm{mm}$ culture dishes for $24 \mathrm{~h}$. The culture medium was replaced with five types of media, as aforementioned. Cells were treated with $10 \mu \mathrm{M}$ DCFH-DA for $30 \mathrm{~min}$ in the dark, washed once with PBS, collected by centrifugation $(200 \mathrm{x} \mathrm{g})$ for $5 \mathrm{~min}$ at room temperature, and were subsequently suspended in PBS. Intracellular ROS levels indicated by the fluorescence of dichlorofluorescein (DCF) were evaluated by excitation at $488 \mathrm{~nm}$ and measured through a 530/22-nm barrier filter using a Becton-Dickinson FACScan flow cytometer (BD Biosciences, San Diego, CA, USA) (7). The data were acquired, analyzed and plotted by the CellQuest Pro software (BD Biosciences) and the SigmaPlot 10.0 software (Systate Software, Inc., San Jose, CA, USA).

Western blot analysis. The MDCK $\left(4 \times 10^{5}\right)$ cells were plated in $100-\mathrm{mm}$ culture dishes for $24 \mathrm{~h}$. The culture medium was replaced with five types of media, as aforementioned. Following treatment, the cells were washed with PBS, resuspended in a protein extraction buffer for $10 \mathrm{~min}$, and centrifuged at $12,000 \mathrm{xg}$ for $10 \mathrm{~min}$ at $4^{\circ} \mathrm{C}$ to obtain the total extracted proteins (in the supernatant). Protein concentrations were measured with a Bio-Rad protein assay reagent (Bio-Rad, Richmond, CA, USA). The extracted cellular proteins were boiled in loading buffer, and an aliquot corresponding to 60-100 $\mu \mathrm{g}$ of protein was separated on a $12 \%$ sodium dodecyl sulfate (SDS)-polyacrylamide gel. Following electrophoresis, proteins were electrotransferred onto a polyvinylidene fluoride transfer membrane. Following blotting, the membranes were incubated with various primary antibodies overnight [p47phox polyclonal antibody (rabbit anti-human; 1:1,000 dilution; cat.no.sc-14015; SantaCruzBiotechnology,Inc.),p22 polyclonal antibody (rabbit anti-human; 1:500 dilution; cat. no. sc-20781; Santa Cruz Biotechnology, Inc.), $\gamma$-GCS polyclonal antibody (rabbit anti-human; 1:750 dilution; cat. no. sc-22755; Santa Cruz Biotechnology, Inc.), SOD-1 monoclonal antibody (mouse anti-human; 1:750 dilution; cat. no. sc-17767; Santa Cruz Biotechnology, Inc.), GAPDH polyclonal antibody (rabbit anti-human; 1:5,000 dilution; cat. no. sc-25778; Santa Cruz Biotechnology, Inc.), catalase polyclonal antibody (rabbit anti-human; 1:5,000 dilution; cat. no. 219010; Merck Millipore Corporation) or $\beta$-actin polyclonal antibody (rabbit anti-human; 1:1,000 dilution; cat. no. A5060; Sigma-Aldrich Co.)] and were washed with PBST solution $0.05 \%$ Tween-20 in PBS (PBST). Subsequent to washing, the secondary antibodies (goat anti-rabbit; 1:10,000 dilution; cat. no. sc-2004; or goat anti-mouse; 1:5,000 dilution; cat. no. sc-2005; Santa Cruz Biotechnology, Inc.) labeled with horseradish-peroxidase was added to the membrane for $1 \mathrm{~h}$ and the sample was washed with PBST solution. The antigen-antibody complexes were detected by enhanced chemiluminescence (Amersham Pharmacia Biotech, Piscataway, NJ, USA) with a chemiluminescence analyzer.

Statistical analysis. Data are presented as the mean \pm standard deviation of at least 3 independent experiments and were analyzed using Student's t-test by the Sigma Plot 10.0 software. $\mathrm{P}<0.05$ was considered to indicate a statistically significant difference.

\section{Results}

Antioxidant activity of Re and Ge in HG treatment. The antioxidant activity of Re and Ge in HG treatment was first evaluated in MDCK cells by DCFH-DA staining and flow cytometry. The DCFH-DA is a probe for intracellular ROS. 
A

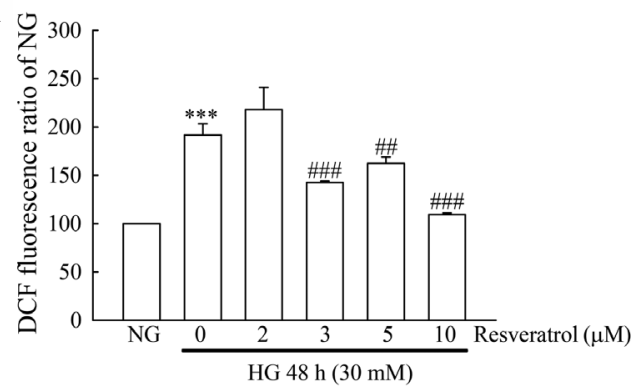

B

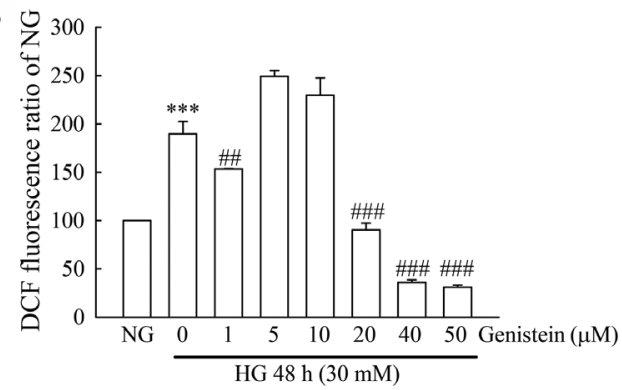

Figure 1. Evaluation of intracellular ROS in resveratrol- or genistein-treated HG-incubated MDCK cells. MDCK cells $\left(2 \times 10^{5}\right)$ were plated in $60-\mathrm{mm}$ cultured dishes for $24 \mathrm{~h}$. MDCK cells were separately grown under NG and $\mathrm{HC}$ media for $48 \mathrm{~h}$. Except for the NG and HG groups, cells in the HG incubation were treated with or without (A) resveratrol $(2,3,5$ and $10 \mu \mathrm{M})$ or (B) genistein $(1,5,10,20,40$ and $50 \mu \mathrm{M})$. Following treatment, the intracellular ROS production was detected by flow cytometry using DCFH-DA staining. The values shown are mean \pm standard deviation ( $\mathrm{n}=5-8$ samples/experiment). Significant differences from the NG group are ${ }^{* * *} \mathrm{P}<0.001$ and the HG group are ${ }^{\# \#} \mathrm{P}<0.01$ and ${ }^{\# \# \#} \mathrm{P}<0.001$, respectively. ROS, reactive oxygen species; $\mathrm{HG}$, high glucose; MDCK, Madin-Darby canine kidney; NG, normal glucose; DCFH-DA, dichlorofluorescein diacetate.

In Fig. 1, 30 mM glucose (HG) incubation induced a 1.7-fold intracellular ROS increase, as compared with $5.5 \mathrm{mM}$ glucose (NG) treatment. $\operatorname{Re}(3,5$ and $10 \mu \mathrm{M})$ treatment led to a significant inhibition of intracellular ROS as compared with the HG treatment. The significant inhibition of intracellular ROS appeared following 1, 20, 40 and $50 \mu \mathrm{M}$ Ge treatment, but not in 5 and $10 \mu \mathrm{M}$ of Ge treatment. To evaluate the synergistic antioxidant activity in Re and Ge, $3 \mu \mathrm{M}$ of Re and $1 \mu \mathrm{M}$ of $\mathrm{Ge}$ were selected for the further experiments.

Re with Ge synergistically inhibits intracellular ROS in $H G$ treatment. The antioxidant activity of Re alone, Ge alone and the Re/Ge combination in HG-treated MDCK cells was evaluated by DCFH-DA staining and flow cytometry. Glucose (HG) of $30 \mathrm{mM}$ of treatment induced a 1.7-fold intracellular ROS as compared with $5.5 \mathrm{mM}$ glucose (NG) treatment (Fig. 2). Re alone or Ge alone led to significant inhibition of intracellular ROS as compared with HG treatment. Of note, the intracellular ROS was significantly inhibited in the combined administration of $\mathrm{Re}$ and $\mathrm{Ge}$, as compared with a single compound treatment.

Multiple sources of ROS are induced by HG treatment in $M D C K$ cells. To evaluate the ROS sources of HG-treated MDCK cells, cells were pre-incubated with $20 \mathrm{nM}$ RO (a complex I inhibitor), $5 \mu \mathrm{M}$ TTFA (a complex II inhibitor), $5 \mu \mathrm{M}$ Car (a complex II inhibitor), $0.1 \mathrm{nM}$ AA (a complex III

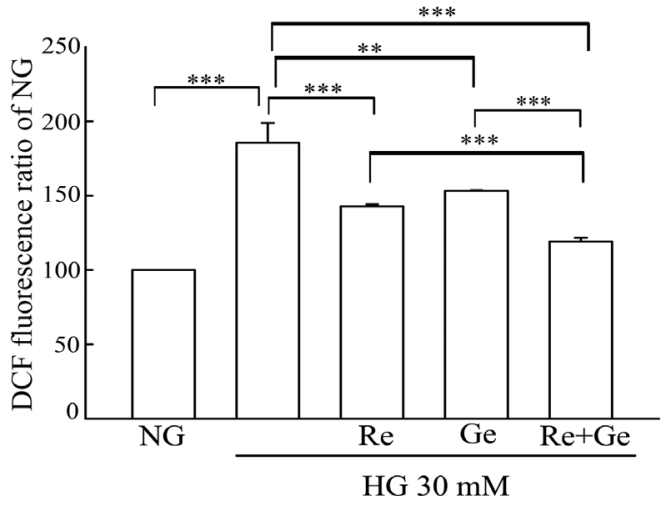

Figure 2. Evaluation of intracellular ROS in the Re/Ge combination-treated HG-incubated MDCK cells. MDCK cells $\left(2 \times 10^{5}\right)$ were plated in $60-\mathrm{mm}$ cultured dishes for $24 \mathrm{~h}$. MDCK cells were separately grown under NG and $\mathrm{HG}$ media for $48 \mathrm{~h}$. Except for the NG and HG groups, cells in the HG incubation were treated with or without $3 \mu \mathrm{M}$ Re alone, $1 \mu \mathrm{M}$ Ge alone, and the $\mathrm{Re} / \mathrm{Ge}$ combination. Following treatment, intracellular ROS production was detected by flow cytometry using DCFH-DA staining. The values shown are mean \pm standard deviation $\left(\mathrm{n}=5-8\right.$ samples/experiment). ${ }^{* *} \mathrm{P}<0.01$ and ${ }^{* * *} \mathrm{P}<0.001$. ROS, reactive oxygen species; Re, resveratrol; Ge, genistein; HG, high glucose; MDCK, Madin-Darby canine kidney; NG, normal glucose; DCFH-DA, dichlorofluorescein diacetate.

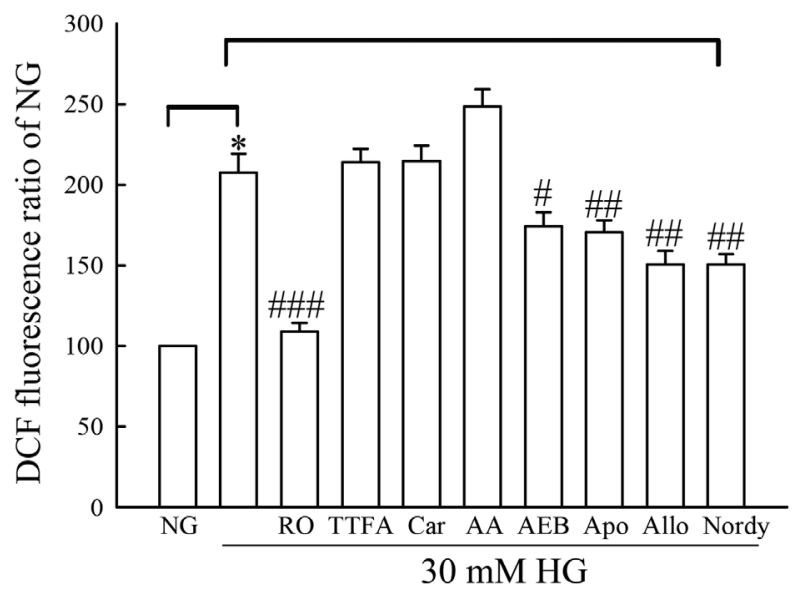

Figure 3. Identification of the originating sites of HG-induced ROS overproduction using a group of specific inhibitors. MDCK cells $\left(2 \times 10^{5}\right)$ were plated in $60-\mathrm{mm}$ cultured dishes for $24 \mathrm{~h}$. MDCK cells were separately grown under NG and HG media for $48 \mathrm{~h}$. Except for the NG and HG groups, cells in the HG group were pretreated with or without various concentrations of designated inhibitors including $20 \mathrm{nM}$ rotenone (RO; mitochondrial complex I), $5 \mu \mathrm{M}$ of 2-thenoyltrifluoroacetone (TTFA; mitochondrial complex II), $5 \mu \mathrm{M}$ carboxin (Car; mitochondrial complex II), $0.01 \mathrm{nM}$ antimycin A (AA; mitochondrial complex III), $1 \mu \mathrm{M}$ 4-(2-aminoethyl)benzenesulfonyl fluoride hydrochloride (AEB; NADPH oxidase), $30 \mu \mathrm{M}$ apocynin (Apo; NADPH oxidase), $20 \mu \mathrm{M}$ allopurinol (Allo; xanthine oxidase) and $5 \mu \mathrm{M}$ nordydihydroguaiaretic acid (Nordy; lipoxygenase) for $1 \mathrm{~h}$. Intracellular ROS production was detected by flow cytometry using DCFH-DA staining. The values shown are mean \pm standard deviation $(\mathrm{n}=5-8$ samples/experiment). Significant differences from the NG group are ${ }^{*} \mathrm{P}<0.05$ and the $\mathrm{HG}$ group are ${ }^{\#} \mathrm{P}<0.05,{ }^{\# \#} \mathrm{P}<0.01$ and ${ }^{\# \# \#} \mathrm{P}<0.001$, respectively. ROS, reactive oxygen species; HG, high glucose; MDCK, Madin-Darby canine kidney; NG, normal glucose; DCFH-DA, dichlorofluorescein diacetate.

inhibitor), $2 \mu \mathrm{M}$ AEB (a NADPH oxidase inhibitor), $30 \mu \mathrm{M}$ Apo (an NADPH oxidase inhibitor), $10 \mu \mathrm{M}$ Allo (a xanthine oxidase inhibitor) or $5 \mu \mathrm{M}$ Nordy (a lipoxygenase inhibitor) for $1 \mathrm{~h}$, followed by co-incubation with $30 \mathrm{mM}$ glucose (HG) for $48 \mathrm{~h}$. Following treatment, the intracellular ROS production 


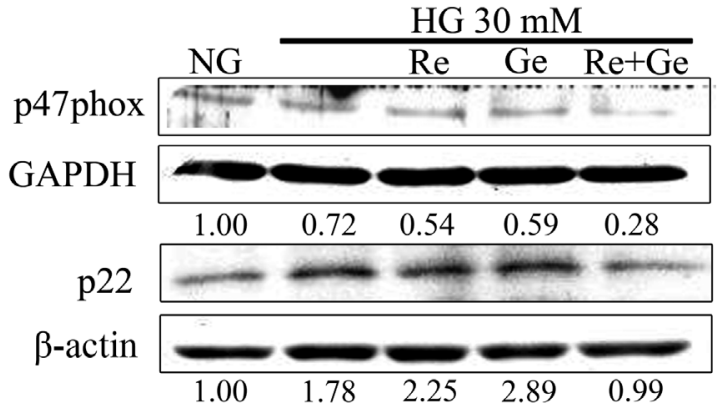

Figure 4. Expression of NADPH oxidase subunits in Re/Ge combination-treated HG-incubated MDCK cells. MDCK cells $\left(4 \times 10^{5}\right)$ were plated in $100-\mathrm{mm}$ cultured dishes for $24 \mathrm{~h}$. MDCK cells were separately grown under NG and $\mathrm{HG}$ media for $48 \mathrm{~h}$. Except for the NG and HG groups, cells in the $\mathrm{HG}$ incubation were treated with or without $3 \mu \mathrm{M}$ Re alone, $1 \mu \mathrm{M} \mathrm{Ge}$ alone, and $\mathrm{Re} / \mathrm{Ge}$ combination. Following treatment, the expression levels of the NADPH oxidase subunits, p47phox and p22, were detected using western blotting. NADPH, nicotinamide adenine dinucleotide phosphate; Re, resveratrol; Ge, genistein; HG, high glucose; NG, normal glucose; MDCK, Madin-Darby canine kidney.

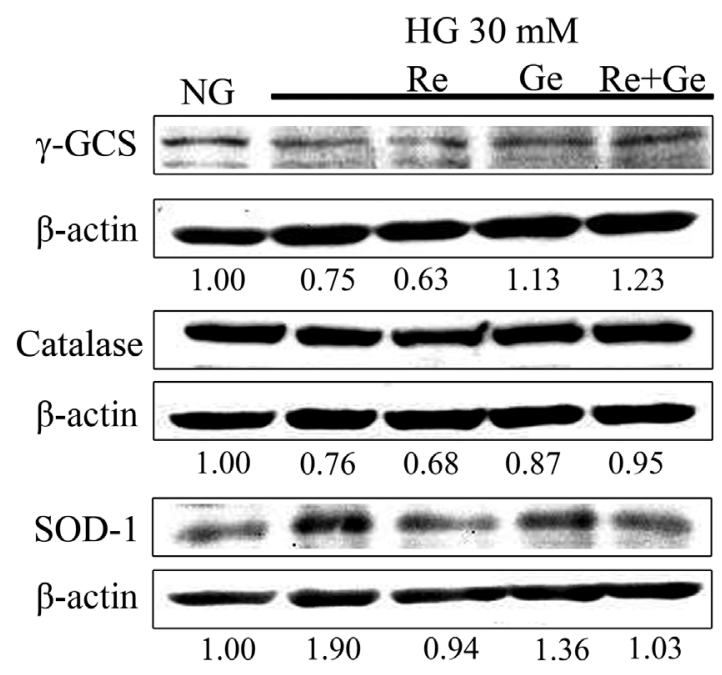

Figure 5. Expression of antioxidant enzymes in the Re/Ge combination-treated HG-incubated MDCK cells. MDCK cells $\left(4 \times 10^{5}\right)$ were plated in $100-\mathrm{mm}$ cultured dishes for $24 \mathrm{~h}$. MDCK cells were separately grown under NG and HG media for $48 \mathrm{~h}$. Except for the NG and HG groups, cells in the HG incubation were treated with or without $3 \mu \mathrm{M}$ Re alone, $1 \mu \mathrm{M}$ Ge alone and the $\mathrm{Re} / \mathrm{Ge}$ combination. Following treatment, the expression levels of the antioxidant enzymes, $\gamma$-GCS, catalase and SOD-1, were detected using western blotting. Re, resveratrol; Ge, genistein; $\mathrm{HG}$, high glucose; NG, normal glucose; MDCK, Madin-Darby canine kidney; $\gamma$-GCS, $\gamma$-glutamylcysteine synthetase; SOD-1, superoxide dismutase-1.

was measured using the DCFH-DA probe. A significant increase in intracellular ROS following HG treatment was observed in the MDCK cells (Fig. 3). The HG-induced intracellular ROS produced, measured using the DCFH-DA probe and flow cytometry, was significantly scavenged when cells were pre-incubated for $1 \mathrm{~h}$ with RO, AEB, Apo, Allo and Nordy in the MDCK cells (Fig. 3). However, TTFA, Car and AA did not scavenge the HG-induced intracellular ROS. These results indicated that there were 4 main sources of ROS, including mitochondrial complex I, NADPH oxidase, xanthine oxidase and lipoxygenase, induced by HG incubation in MDCK cells.

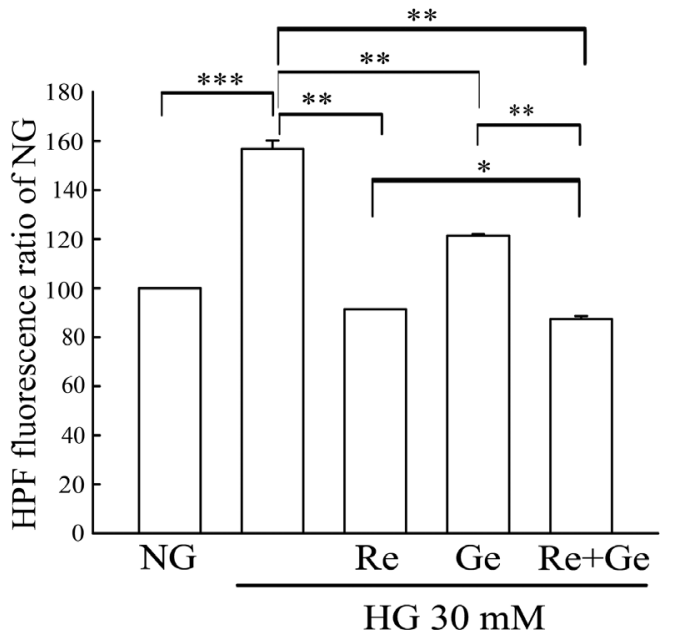

Figure 6. Evaluation of the intracellular hydroxyl radicals in the $\mathrm{Re} / \mathrm{Ge}$ combination-treated HG-incubated MDCK cells. MDCK cells $\left(2 \times 10^{5}\right)$ were plated in $60-\mathrm{mm}$ cultured dishes for $24 \mathrm{~h}$. MDCK cells were separately grown under $\mathrm{NG}$ and $\mathrm{HG}$ media for $48 \mathrm{~h}$. Except for the $\mathrm{NG}$ and $\mathrm{HG}$ groups, cells in the $\mathrm{HG}$ incubation were treated with or without $3 \mu \mathrm{M}$ Re alone, $1 \mu \mathrm{M} \mathrm{Ge}$ alone and the $\mathrm{Re} / \mathrm{Ge}$ combination. Following treatment, intracellular reactive oxygen species production was detected by flow cytometry using HPF staining. The values shown are mean \pm standard deviation $(\mathrm{n}=5-8$ samples/experiment). ${ }^{*} \mathrm{P}<0.05,{ }^{* *} \mathrm{P}<0.01$ and ${ }^{* * *} \mathrm{P}<0.001$, respectively. Re, resveratrol; Ge, genistein; HG, high glucose; NG, normal glucose; MDCK, Madin-Darby canine kidney; HPF, hydroxyphenyl fluorescein.

Re/Ge synergistically inhibits NADPH oxidase expression in $H G$ treatment. NADPH oxidase is a main source of intracellular ROS production. The intracellular ROS produced by HG treatment also involved NADPH oxidase. The expression of two subunits of NADPH oxidase, p47phox and p22, were further evaluated in $\mathrm{HG}$ incubation. In Fig. 4, the expression of p22 was increased to 1.78-fold in HG-treated cells. Of note, Re or Ge alone treatment led to a 2.25- and 2.89-fold increase of p22 expression. There was no increased expression following treatment with the Re/Ge combination in the HG-treated cells (only 0.99-fold p22). Treatment with Re or Ge alone decreased the p47phox expression to 0.54- and 0.59-fold of the NG treatment. The Re/Ge combination caused more inhibition of p47phox expression, which was 0.28 -fold of the NG incubation.

Re with Ge synergistically effects the expression of antioxidant enzymes in HG treatment. $\gamma$-GCS, catalase and SOD-1 are three critical antioxidant enzymes, which act against cellular ROS during oxidative stress. To evaluate the expression of these antioxidant enzymes in HG incubation, western blotting was used. The expression of $\gamma$-GCS decreased to 0.75 -fold in the HG treatment as compared with NG incubation (Fig. 5). The expression level of $\gamma$-GCS following treatment with Re alone in HG incubation was decreased to 0.63 -fold. However, an increase to 1.13-fold was observed in the expression of $\gamma$-GCS when MDCK cells were treated with Ge alone in $\mathrm{HG}$ for $48 \mathrm{~h}$. Treatment with the Re/Ge combination in HG-treated cells for $48 \mathrm{~h}$ further increased the $\gamma$-GCS expression to 1.23-fold as compared with NG incubation. The data appear to indicate that the increased expression of $\gamma$-GCS for the Re/Ge combination treatment in HG-treated cells is an important event for ROS elimination. The catalase expression was markedly decreased to 0.76 - and 0.68 -fold in HG incubation and 
Re alone treatment, respectively. Notably, catalase expression was 0.95 -fold in the $\mathrm{Re} / \mathrm{Ge}$ combination treatment in the $\mathrm{HG}$ incubation. The expression of SOD-1 in MDCK cells was increased to 1.9-fold in the HG treatment at $48 \mathrm{~h}$; however, the $\mathrm{Re} / \mathrm{Ge}$ combination in the HG incubation resulted in 1.03-fold of SOD-1 expression as compared with NG incubation.

Re/Ge synergistically inhibits intracellular hydroxyl radials in $H G$ treatment. The antioxidant activity on hydroxyl radicals of Re alone, Ge alone and Re/Ge combination in the HG-treated MDCK cells was evaluated by HPF staining and flow cytometry. HG incubation induced a 1.5-fold increase in intracellular hydroxyl radicals as compared with NG incubation (Fig. 6). Re alone or Ge alone led to a significant inhibition of intracellular hydroxyl radicals as compared with $\mathrm{HG}$ incubation. Of note, the intracellular hydroxyl radicals were significantly inhibited in the $\mathrm{Re} / \mathrm{Ge}$ combination as compared with $\mathrm{Re}$ or Ge alone treatment.

\section{Discussion}

In Fig. 3, RO (a complex I inhibitor) inhibited the largest amount of ROS in the HG treatment, suggesting the site of the ROS source provided from HG incubation is mitochondrial complex I. Previous studies have indicated that the hyperglycemia characteristics of diabetes increase the complex I substrate NADH (8), which is likely to potentiate ROS production by the respiratory chain (9). This is in agreement with the present finding that mitochondrial complex I generated the maximum amount of ROS in the HG-treated MDCK cells. An important source of ROS production is NADPH oxidase. This enzyme results in superoxides during the respiratory burst in phagocytes. The NADPH oxidase consists of membrane-bound subunits (gp91phox and p22phox) and cytosolic subunits (p47phox, p40phox, p67phox and Rac) (10). When activated, p47phox is phosphorylated, and the cytosolic components translocate to the membrane, where they form a molecular cluster of the catalytically active oxidase. The expression of p47phox appeared to exhibit the largest inhibition by the $\mathrm{Re} / \mathrm{Ge}$ combination as compared with $\mathrm{Re}$ or Ge alone, indicating that the Ge/Re combination inhibits the activation of NADPH oxidase in HG incubation.

Xanthine oxidase is an enzyme that converts hypoxanthine/xanthine to uric acid in a reaction that liberates superoxide. High activity of xanthine oxidase has been observed in numerous pathological conditions, as the xanthine oxidase-generated superoxide is cytotoxic. A significant increase in the activity of xanthine oxidase was observed in diabetics (11). Inhibition of xanthine oxidase reduces hyperglycemia-induced oxidase stress in skeletal muscle of diabetic mice. Evidence is accumulating that some of the products derived from the actions of lipoxygenases contribute to diabetic nephropathy. Specifically, levels of lipoxygenases 12 and 15 are increased in diabetic animals. In addition, HG levels increase the expression of lipoxygenases 12 and 15 in cultured mesangial cells (12). The results of the present study show that Allo (a xanthine oxidase inhibitor) and Nordy (a lipooxygenase inhibitor) inhibited a partial ROS level in HG-incubated MDCK cells (Fig. 3) suggesting that xanthine oxidase and lipoxygenase also have important roles in ROS generation of kidney cells in diabetes.
Other possible mechanisms by which $\mathrm{HG}$ incubation increases intracellular ROS is the upregulation of SOD-1 observed in MDCK cells (Fig. 5). SOD-1 is a superoxide metabolic enzyme that metabolizes superoxide to $\mathrm{H}_{2} \mathrm{O}_{2}$, which is further metabolized to $\mathrm{H}_{2} \mathrm{O}$ and $\mathrm{O}_{2}$ by catalase. $\mathrm{HG}$ incubation markedly increased the expression of SOD-1, and this effect would result in intracellular superoxide being converted to $\mathrm{H}_{2} \mathrm{O}_{2}$ (Fig. 5). Furthermore, as $\mathrm{HG}$ incubation significantly inhibited the expression of catalase to 0.76-fold (Fig. 5), the elimination of intracellular $\mathrm{H}_{2} \mathrm{O}_{2}$ may not be executed normally in MDCK cells and may ultimately contribute to the increase in intracellular $\mathrm{H}_{2} \mathrm{O}_{2}$ after $48 \mathrm{~h}$ of $\mathrm{HG}$ incubation. By contrast, the $\mathrm{Re} / \mathrm{Ge}$ combination could recover the expression of catalase to 0.95 -fold in $\mathrm{HG}$ incubation suggesting that the synergistic antioxidant activity is partially a result from the maintenance of catalase expression.

$\gamma$-GCS is the first enzyme of the cellular glutathione (GSH) biosynthetic pathway that catalyzes the chemical reaction. GSH combines with hydroxyl radical, peroxynitrite, and hydroperoxides, as well as reactive electrophiles, including activated phosphoramide mustard (13). The activity of $\gamma$-GCS was significantly lower in diabetics compared to normal controls (14). A decreased expression of $\gamma$-GCS in diabetic rats is consistent with repressed GSH synthesis (15). These results are in agreement with the present findings that the expression of $\gamma$-GCS in HG incubation was decreased to 0.75 -fold of NG-incubated cells. The expression of $\gamma$-GCS was increased to 1.23-fold in the Re/Ge combination indicating another important antioxidant mechanism of the Re/Ge combination, which provides an enhanced GSH synthesis function to antagonize the ROS in HG incubation.

Re has potent protective effects on diabetes and diabetic complications including diabetic nephropathy. Xu et al (16) demonstrated that pretreatment with $\operatorname{Re}(10 \mu \mathrm{M})$ for $6 \mathrm{~h}$ prior to $\mathrm{HG}(30 \mathrm{mM})$ treatment for $48 \mathrm{~h}$ significantly reduced the hyperglycemia-induced increase in ROS production in rat mesangial cells. This is in agreement with the present results, as $\operatorname{Re}(10 \mu \mathrm{M})$ alone decreased HG-induced ROS in MDCK cells. In addition, another study also demonstrated that the addition of Ge to soy protein causes improvements in the antioxidant status of kidney tissue. The catalase activity was significantly increased in soy/Ge treatment (17). The present findings explain the observation that the low concentration of $\mathrm{Re}$ $(3 \mu \mathrm{M})$ in combination with Ge $(1 \mu \mathrm{M})$ could further decrease the HG-induced ROS, suggesting that an important synergistic antioxidant effect may be applied in diabetic nephropathy.

\section{Acknowledgements}

The present study was supported by a grant from the Ministry of Economic Affairs of Taiwan, R.O.C(no. 104-EC-17-A-18-S1-226).

\section{References}

1. Tisminetzky M, McManus DD, Dor A, Miozzo R, Yarzebski J, Gore JM and Goldberg RJ: Decade-long trends (1999-2009) in the characteristics, management, and hospital outcomes of patients hospitalized with acute myocardial infarction with prior diabetes and chronic kidney disease. Int J Nephrol Renovasc Dis 8: 41-51, 2015.

2. Kashihara N, Haruna Y, Kondeti VK and Kanwar YS: Oxidative stress in diabetic nephropathy. Curr Med Chem 17: 4256-4269, 2010. 
3. de la Lastra CA and Villegas I: Resveratrol as an antioxidant and pro-oxidant agent: mechanisms and clinical implications. Biochem Soc Trans: 35, 1156-1160, 2007.

4. Han RM, Tian YX, Liu Y, Chen CH, Ai XC, Zhang JP and Skibsted LH: Comparison of flavonoids and isoflavonoids as antioxidants. J Agric Food Chem 57: 3780-3785, 2009.

5. Borrás C, Gambini J, López-Grueso R, Pallardó FV and Viña J: Direct antioxidant and protective effect of estradiol on isolated mitochondria. Biochim Biophys Acta 1802: 205-211, 2010.

6. Ullmann K, Wiencierz AM, Müller C, Thierbach R, Steege A, Toyokuni S and Steinberg P: A high-throughput reporter gene assay to prove the ability of natural compounds to modulate glutathione peroxidase, superoxide dismutase and catalase gene promoters in V79 cells. Free Radic Res 42: 746-753, 2008.

7. Yang JT, Li ZL, Wu JY, Lu FJ and Chen CH: An oxidative stress mechanism of shikonin in human glioma cells. PLoS One 9: e94180, 2014.

8. Kabat A, Pönicke K, Salameh A, Mohr FW and Dhein S: Effect of a beta 2-adrenoceptor stimulation on hyperglycemia-induced endothelial dysfunction. J Pharmacol Exp Ther 308: 564-573, 2004

9. Brownlee M: Biochemistry and molecular cell biology of diabetic complications. Nature 414: 813-820, 2001.

10. Babior BM: NADPH oxidase. Curr Opin Immunol 16: 42-47, 2004
11. Suriyajothi MA, Sangeetha R and Venkateswari R: Activity of xanthine oxidase of diabetes: Its correlation with aging. Pharmacologyonline 2: 128-133, 2011.

12. Hao CM and Breyer MD: Roles of lipid mediators in kidney injury. Semin Nephrol 27: 338-351, 2007.

13. Griffith OW and Mulcahy RT: The enzymes of glutathione synthesis: Gamma-glutamylcysteine synthetase. Adv Enzymol Relat Areas Mol Biol 73: 209-267, xii, 1999.

14. Murakami K: Glutathione metabolism in erythrocytes from patients with diabetes mellitus. Hokkaido Igaku Zasshi 66: 29-40, 1991 (In Japanese).

15. Furfaro AL, Nitti M, Marengo B, Domenicotti C, Cottalasso D, Marinari UM, Pronzato MA and Traverso N: Impaired synthesis contributes to diabetes-induced decrease in liver glutathione. Int J Mol Med 29: 899-905, 2012.

16. Xu Y, Nie L, Yin YG, Tang JL, Zhou JY, Li DD and Zhou SW: Resveratrol protects against hyperglycemia-induced oxidative damage to mitochondria by activating SIRT1 in rat mesangial cells. Toxicol Appl Pharmacol 259: 395-401, 2012.

17. Javanbakht MH, Sadria R, Djalali M, Derakhshanian H, Hosseinzadeh P, Zarei M, Azizi G, Sedaghat R and Mirshafiey A: Soy protein and genistein improves renal antioxidant status in experimental nephrotic syndrome. Nefrologia 34: 483-490, 2014. 